MATHEMATICS OF COMPUTATION

Volume 69, Number 230, Pages 443-462

S 0025-5718(99)01085-6

Article electronically published on February 24, 1999

\title{
CAN A FINITE ELEMENT METHOD PERFORM ARBITRARILY BADLY?
}

\author{
IVO BABUŠKA AND JOHN E. OSBORN
}

\begin{abstract}
In this paper we construct elliptic boundary value problems whose standard finite element approximations converge arbitrarily slowly in the energy norm, and show that adaptive procedures cannot improve this slow convergence. We also show that the $L_{2}$-norm and the nodal point errors converge arbitrarily slowly. With the $L_{2}$-norm two cases need to be distinguished, and the usual duality principle does not characterize the error completely. The constructed elliptic problems are one dimensional.
\end{abstract}

\section{INTRODUCTION}

The classical finite element method approximates the exact solution $u$ of an elliptic boundary value problem by piecewise polynomials (or pull-back polynomials when curvilinear elements are used). Denoting by $u_{N}$ the finite element approximation with $N$ degrees of freedom (i.e., the approximate solution for which $N$ unknowns have to be determined), for reasonable meshes we have $\left\|u-u_{N}\right\|_{E} \rightarrow 0$ $\left(\|\cdot\|_{E}\right.$ denotes the energy norm) as $N \rightarrow \infty$, provided $u \in H^{1}$. If $u$ has additional smoothness, then typically $\left\|u-u_{N}\right\|_{E} \leq C N^{\frac{-\mu}{n}}$, where $\mu$ depends on the smoothness of $u$ and the degree of the elements, and $n$ is the dimension of the problem. If the solution $u$ has additional properties, e.g., $u$ is the solution of Laplace's equation in a polygonal or polyhedral domain and is singular at the corners or edges, and the mesh is properly selected a priori or by an adaptive procedure, then we typically have $\left\|u-u_{N}\right\|_{E} \leq C N^{\frac{-p}{n}}$, where $p$ is the degree of the elements.

Hence the question arises whether that there are problems for which the classical finite element method converges arbitrarily slowly. We show that there are such problems, and furthermore that the convergence cannot be improved with adaptivity. Specifically, we show that given a sequence of nonincreasing positive numbers $\chi_{N}$, with $\chi_{1}=1$, that converges to 0 , there is a problem with solution $u \in H^{1}$ such that

- there are constants $C_{1}$ and $C_{2}$, independent of $\left\{\chi_{N}\right\}$, such that

$$
C_{1} \chi_{N} \leq\left\|u-u_{N}\right\|_{E} \leq C_{2} \chi_{N} \text {, for all } N
$$

(briefly, the energy norm error is of order $\chi_{N}$ );

- there is a sequence $1 \leq N_{1}<N_{2}<\cdots$ such that $\left\|u-u_{N_{i}}\right\|_{L_{2}}$ is the order of $\chi_{N_{i}}^{2}$, provided $\chi_{N}$ converges to 0 "very slowly" (in a sense to be made

Received by the editor May 5, 1998.

1991 Mathematics Subject Classification. Primary: 65N15, 65N30.

Key words and phrases. Finite element methods, convergence, adaptivity, rough coefficients.

The first author was supported in part by NSF Grant \#DMS-95-01841.

(C)2000 American Mathematical Society 
more precise later); the usual duality principle does not characterize the error completely; and

- the nodal point errors are the order of $\chi_{N}^{2}$.

These results are proved for a family of uniform meshes. We also show that the errors behave in the same way for adaptively constructed meshes.

The example we construct is a one-dimensional boundary value problem, with a homogeneous differential equation, a homogeneous Dirichlet condition at one end, and a non-homogeneous Neumann condition at the other end. The differential equation has a rough coefficient $a(x)$, satisfying $0<\alpha \leq a(x) \leq \beta$, which is constructed in terms of the sequence $\left\{\chi_{N}\right\}$. We expect that the results we obtain also hold for general boundary value problems: boundary value problems in one or more dimensions, with general Dirichlet, Neumann, or mixed Dirichlet/Neumann boundary conditions.

Problems with rough coefficients are typical in problems with heterogeneous materials. The example we construct shows that finite element methods based on piecewise polynomial elements lead to unacceptable results when applied to these problems. In addition, it shows that the comparison of results computed with coarser and finer meshes cannot be used as a basis for assessing the accuracy of the results. This example also shows the importance of developing special methods for these problems; the special element method introduced and analyzed in [2, 5] is an example of such a method.

Section 2 describes the specific boundary value problem we consider. In Section 3 we construct the specific coefficient $a(x)$, and state and prove the estimates for the energy norm of the error. The estimates for the nodal point errors are proved in Section 4. In Section 5, the $L_{2}$-error is analyzed. Remark 7, in Section 5, discusses the relation of the $L_{2}$-error to $L_{2}$-error estimates proved with the usual duality argument. Remark 8 , in Section 5 , discusses the error in the $L_{\infty}$-norm and superconvergence at the nodes. Section 6 discusses adaptively constructed meshes for our example. Section 7 summarizes our conclusions.

\section{A Model Boundary VALUE PROBLem AND ITS FINITE ELEMENT APPROXIMATION}

We consider the specific model boundary value problem

$$
\left\{\begin{array}{l}
-\frac{d}{d x}\left(a(x) \frac{d u}{d x}\right)=0, \quad 0<x<1, \\
u(0)=0, \quad a u^{\prime}(1)=1,
\end{array}\right.
$$

where $a(x)$ is a measurable function on $I=(0,1)$ satisfying

$$
0<\alpha \leq a(x) \leq \beta .
$$

The solution $u(x)$ of (2.1) can be interpreted as the longitudinal displacement of a heterogeneous bar with (local) modulus of elasticity $a(x)$ that is subject to no longitudinal load, with left end fixed, and with the stress at the right end equal to 1 .

We will understand (2.1) in the weak or variational sense:

$$
\left\{\begin{array}{l}
u \in H_{0^{l}}^{1}(I), \\
B(u, v)=\int_{0}^{1} a(x) u^{\prime} v^{\prime} d x=v(1), \quad \forall v \in H_{0^{l}}^{1}(I),
\end{array}\right.
$$


where

$$
H^{1}(I)=\left\{u:\|u\|_{H^{1}(I)}^{2}=\int_{0}^{1}\left[\left(u^{\prime}\right)^{2}+u^{2}\right] d x<\infty\right\}
$$

and

$$
H_{0^{l}}^{1}(I)=\left\{u \in H^{1}(I): u(0)=0\right\} .
$$

(The superscript $l$ indicates that the functions in $H_{0^{l}}^{1}(I)$ are required to be 0 at the left endpoint of the interval $I$.) The solution of (2.3), or (2.1), exists and is unique, and is given by

$$
u(x)=\int_{0}^{x} \frac{d t}{a(t)} .
$$

Sometimes we will indicate the dependence of $u$ on the coefficient $a$ by writing $u_{a}$.

On $H_{0^{l}}^{1}(I)$ we will also use the energy norm

$$
\|u\|_{E}=[B(u, u)]^{1 / 2}=\left[\int_{0}^{1} a\left(u^{\prime}\right)^{2} d x\right]^{1 / 2}
$$

and the norm

$$
|u|_{H^{1}(I)}=\left[\int_{0}^{1}\left(u^{\prime}\right)^{2} d x\right]^{1 / 2}
$$

$\|u\|_{E}$ and $|u|_{H^{1}(I)}$ are equivalent on $H_{0^{l}}^{1}$ :

$$
\sqrt{\alpha}|u|_{H^{1}} \leq\|u\|_{E} \leq \sqrt{\beta}|u|_{H^{1}} .
$$

We will also use the $L_{2}(I)$ - and $L_{\infty}(I)$-norms.

We are interested in the approximation of the solution of (2.3) by a usual (polynomial based) finite element method. Toward this end we let $\Delta=\left\{0=x_{0}^{\Delta}<x_{1}^{\Delta}<\right.$ $\left.\cdots<x_{N}^{\Delta}=1\right\}$, where $N=N^{\Delta}$ is a positive integer, be an arbitrary mesh on $I$, and let $I_{j}=I_{j}^{\Delta}=\left(x_{j-1}^{\Delta}, x_{j}^{\Delta}\right), j=1, \ldots, N$, and $h=h^{\Delta}=\max _{1 \leq j \leq N}\left(x_{j}^{\Delta}-x_{j-1}^{\Delta}\right)$. Further, let

$$
\mathcal{V}^{\Delta}=\left\{u \in H_{0^{l}}^{1}(I):\left.u\right|_{I_{j}^{\Delta}} \in \mathcal{P}^{1}\left(I_{j}^{\Delta}\right), j=1, \ldots, N^{\Delta}\right\},
$$

where $\mathcal{P}^{1}\left(I_{j}^{\Delta}\right)$ is the set of polynomials of degree less than or equal to 1 (considered on $I_{j}^{\Delta}$ ), be the associated finite element space. Then, as usual, the corresponding finite element approximation $u^{\Delta}$ to the solution $u$ of (2.3) is characterized by:

$$
\left\{\begin{array}{l}
u^{\Delta} \in \mathcal{V}^{\Delta}, \\
B\left(u^{\Delta}, v\right)=v(1), \quad \forall v \in \mathcal{V}^{\Delta} .
\end{array}\right.
$$

Clearly, $u^{\Delta}$ exists and is unique.

It is known that

$$
\lim _{h^{\Delta} \rightarrow 0}\left\|u-u^{\Delta}\right\|_{E}=0
$$

and hence that

$$
\lim _{h^{\Delta} \rightarrow 0}\left\|u-u^{\Delta}\right\|_{L_{2}(I)}=0 \text { and } \lim _{h^{\Delta} \rightarrow 0}\left\|u-u^{\Delta}\right\|_{L_{\infty}(I)}=0,
$$

provided $a(x)$ satisfies (2.2), and furthermore that $\left\|u-u^{\Delta}\right\|_{E}=O\left(h^{\Delta}\right)$ if $a(x)$ is smooth. We are interested, however, in rough $a(x)$, and in assessing the accuracy of $u^{\Delta}$ for such $a(x)$. We will see that essentially nothing beyond $(2.7 \mathrm{a}, \mathrm{b})$ is true without additional assumptions on $a(x)$. 
Throughout most of the paper we will consider uniform meshes. Specifically, consider the family of uniform meshes given by

$$
\Delta=\Delta_{k}=\left\{x_{k, 0}=\frac{0}{2^{k}}=0<x_{k, 1}=\frac{1}{2^{k}}<\cdots<x_{k, 2^{k}}=\frac{2^{k}}{2^{k}}=1\right\}, \quad k=0,1, \ldots,
$$

and let

$$
\mathcal{V}_{k}=\mathcal{V}^{\Delta_{k}}
$$

Then $N=N^{\Delta_{k}}=2^{k} ; I_{j}=I_{k, j}=I_{j}^{\Delta_{k}}=\left(x_{k, j-1}, x_{k, j}\right), j=1, \ldots, 2^{k} ;$ and $h=$ $h_{k}=h^{\Delta_{k}}=N^{-1}=2^{-k}$. The corresponding finite element approximation $u_{k}$ to $u$ is characterized by

$$
\left\{\begin{array}{l}
u_{k} \in \mathcal{V}_{k} \\
B\left(u_{k}, v\right)=v(1), \quad \forall v \in \mathcal{V}_{k}
\end{array}\right.
$$

cf. (2.6). Note that degrees of freedom $=\operatorname{dim} \mathcal{V}_{k}=N=2^{k}$.

\section{ANALYSis OF THE ERROR IN THE ENERGY NORM}

Let $u$ be the exact solution of the boundary value problem (2.3), or (2.1), and let $u_{k}$ be the finite element approximation determined by the uniform mesh $\Delta_{k}$ with $2^{k}$ elements and $h_{k}=2^{-k}$ described in Section 2. In this section we assess the error in the energy norm.

Theorem 1. Let $\left\{\chi_{k}\right\}_{k=0}^{\infty}$, with $\chi_{0}=1$, be a sequence of nonincreasing positive numbers converging to 0 . Then there is a coefficient $a(x)$ satisfying (2.2) (with $\alpha=0.9$ and $\beta=1.1$ ) such that

$$
\left(3 \cdot 10^{-2}\right) \chi_{k} \leq\left\|u-u_{k}\right\|_{E} \leq 1.9 \chi_{k}, \text { for all } k .
$$

Remark 1. We have chosen to present the constants in our estimates as explicit (decimal) numbers. We have adopted this somewhat unusual practice in part to show that the constants are absolute, in particular that they do on not depend on the sequence $\left\{\chi_{k}\right\}$, and in part to make the proofs of the estimates easier to follow. The coefficient $a(x)$ naturally depends on $\left\{\chi_{k}\right\}$.

Remark 2. The sequence $\left\{\chi_{k}\right\}$ is indexed by $k$ and not by $N=$ degrees of freedom. The result can, however, be recast in terms of $N$ using the relation $N=2^{k}$; $c f$. Section 1. The same is true for Theorems 2 and 3 below.

Proof. Let $u_{k}$ be the approximate solution, as characterized by (2.8). Writing

$$
u_{k}=\sum_{i=1}^{2^{k}} u_{k, i} \phi_{i},
$$


where $\phi_{1}, \ldots, \phi_{2^{k}}$ are the standard basis functions for $\mathcal{V}_{k}\left(\phi_{i} \in \mathcal{V}_{k}, \phi_{i}\left(x_{k, j}\right)=\right.$ $\left.\delta_{i j}, i, j=1, \ldots, 2^{k}\right)$, we easily see from $(2.8)$ that $u_{k, j}=u_{k}\left(x_{k, j}\right)$ satisfy

$$
\left\{\begin{aligned}
\frac{\frac{-\int_{I_{k, j}} a d x}{h_{k}} u_{k, j-1}+\left(\frac{\int_{I_{k, j}} a d x}{h_{k}}+\frac{\int_{I_{k, j+1}} a d x}{h_{k}}\right) u_{k, j}-\frac{\int_{I_{k, j+1}} a d x}{h_{k}} u_{k, j+1}}{h_{k}}=0, & j=1, \ldots, 2^{k}-1, \\
\frac{\frac{-\int_{I_{k, 2^{k}}} a d x}{h_{k}} u_{k, 2^{k}-1}+\frac{\int_{I_{k, 2^{k}}} a d x}{h_{k}} u_{k, 2^{k}}}{h_{k}} & =1 .
\end{aligned}\right.
$$

Letting $a_{k, j}=\frac{\int_{I_{k, j}} a d x}{h_{k}}$ and $z_{k, j}=\frac{\left(u_{k, j}-u_{k, j-1}\right) a_{k, j}}{h_{k}}$, equations (3.2) can be written

$$
z_{k, j}-z_{k, j+1}=0, \quad j=1, \ldots, 2^{k}-1, \quad z_{k, 2^{k}}=1 .
$$

Hence $z_{k, j}=1$ for $j=1, \ldots, 2^{k}$, and equations (3.2) reduce to

$$
\frac{\left(u_{k, j}-u_{k, j-1}\right)}{h_{k}} a_{k, j}=1, \quad j=1, \ldots, 2^{k},
$$

and so, since $u_{k, 0}=0$,

$$
u_{k, j}=u_{k}\left(x_{k, j}\right)=h_{k} \sum_{i=1}^{j} \frac{1}{a_{k, i}} .
$$

Let

$$
\bar{a}_{k, j}=\left(\frac{\int_{I_{k, j}} \frac{d x}{a}}{h_{k}}\right)^{-1}
$$

From (2.4) we see that

$$
\begin{aligned}
u_{j}=u\left(x_{k, j}\right) & =\int_{0}^{x_{k, j}} \frac{d x}{a} \\
& =h_{k}\left\{\frac{\int_{0}^{x_{k, 1}} \frac{d x}{a}}{h_{k}}+\frac{\int_{x_{k, 1}}^{x_{k, 2}} \frac{d x}{a}}{h_{k}}+\cdots+\frac{\int_{x_{k, j-1}}^{x_{k, j}} \frac{d x}{a}}{h_{k}}\right\} \\
& =h_{k} \sum_{i=1}^{j} \frac{1}{\bar{a}_{k, i}} .
\end{aligned}
$$

We will define $a(x)$ in terms of the following $L_{2}(I)$-orthonormal sequence, which is closely related to the Haar basis ( $c f$. [10]):

$$
\begin{gathered}
g_{0}(x)=1, \quad 0<x<1, \\
g_{1}(x)= \begin{cases}1, & 0<x<\frac{1}{2}, \\
-1, & \frac{1}{2}<x<1,\end{cases}
\end{gathered}
$$


and, in general,

$$
g_{l}(x)= \begin{cases}1, & 0<x<\frac{1}{2^{l}}, \\ -1, & \frac{1}{2^{l}}<x<\frac{2}{2^{l}}, \\ 1, & \frac{2}{2^{l}}<x<\frac{3}{2^{l}}, \\ -1, & \frac{3}{2^{l}}<x<\frac{4}{2^{l}}, \\ \vdots & \\ 1, & \frac{2^{l}-2}{2^{2}}<x<\frac{2^{l}-1}{2^{l}}, \\ -1, & \frac{2^{l}-1}{2^{l}}<x<1 .\end{cases}
$$

Let $\gamma=1 / 20$. Then let $0=l_{0}<l_{1}<\cdots$ be inductively selected so that $l_{i+1}$ is the least index $\geq l_{i}+1$ such that

$$
\chi_{l_{i+1}} \leq \gamma \chi_{l_{i}}
$$

It is immediate that

$$
\chi_{l_{(i+1)}-1}>\gamma \chi_{l_{i}}
$$

We then define $a(x)$ by

$$
a(x)=\sum_{l=0}^{\infty} d_{l} g_{l}(x)
$$

where

$$
\begin{aligned}
& d_{l_{0}}=d_{0}=1 ; \\
& d_{l_{i}}=\gamma \chi_{l_{i-1}} \\
& \text { and } d_{l}=0 \quad \text { for } i=1,2, \ldots ;
\end{aligned}
$$

We see immediately that $d_{l_{i}} \leq \gamma^{i} \chi_{0}=\gamma^{i}$, and hence that

$$
\left|\sum_{i=1}^{\infty} d_{l_{i}} g_{l_{i}}(x)\right| \leq \sum_{i=1}^{\infty} \gamma^{i}=\frac{\gamma}{1-\gamma} \leq 0.1
$$

Hence the series defining $a(x)$ converges uniformly and

$$
\alpha=0.9 \leq a(x) \leq 1.1=\beta .
$$

The series defining $a(x)$ is of lacunary, or gap, type.

If $l \leq k$, we see that $g_{l}(x)$ is constant on each $I_{k, j}, j=1, \ldots, 2^{k}$. Hence, if we write

$$
\begin{aligned}
a(x) & =\sum_{l=0}^{k} d_{l} g_{l}(x)+\sum_{l=k+1}^{\infty} d_{l} g_{l}(x) \\
& =\phi_{k}(x)+\eta_{k}(x),
\end{aligned}
$$

we see that $\phi_{k}(x)=\phi_{k, j}$ is constant on each $I_{k, j}, j=1, \ldots, 2^{k}$. Furthermore, if $l>k$, then

$$
\int_{I_{k, j}} g_{l}(x) d x=0, \text { for } j=1, \ldots, 2^{k}
$$

if $l \leq k$, then

$$
\int_{I_{k, j}} g_{l}(x) d x= \pm h_{k}, \text { for } j=1, \ldots, 2^{k}
$$


if $l, m>k, l \neq m$, then

$$
\int_{I_{k, j}} g_{l}(x) g_{m}(x) d x=0, \text { for } j=1, \ldots, 2^{k}
$$

and if $l \geq 0$, then

$$
\int_{I_{k, j}} g_{l}^{2}(x) d x=h_{k}, \text { for } j=1, \ldots, 2^{k} .
$$

From (3.10) and (3.11a,c,d) we see that

$$
\int_{I_{k, j}} \eta_{k}(x) d x=0
$$

and

$$
\int_{I_{k, j}} \eta_{k}(x)^{2} d x=h_{k} \sum_{l=k+1}^{\infty} d_{l}^{2} .
$$

We also have (cf. (3.8) and (3.9))

$$
\alpha=0.9 \leq \phi_{k}(x) \leq 1.1=\beta
$$

and

$$
\left|\eta_{k}(x)\right| \leq 0.1
$$

From (3.10) and (3.12a), recalling that $\phi_{k}(x)=\phi_{k, j}$ is constant on each $I_{k, j}$, we see that $a_{k, j}=\phi_{k, j}$. Thus, from (2.4), (3.3), and (3.10), for $x \in I_{k, j}$ we have

$$
\begin{aligned}
\frac{d u}{d x}-\frac{d u_{k}}{d x} & =\frac{1}{a(x)}-\frac{1}{a_{k, j}} \\
& =\frac{1}{\phi_{k, j}+\eta_{k}(x)}-\frac{1}{\phi_{k, j}} \\
& =\frac{-\eta_{k}(x)}{\phi_{k, j}^{2}\left(1+\frac{\eta_{k}(x)}{\phi_{k, j}}\right)} \\
& =\frac{1}{\phi_{k, j}^{2}}\left(-\eta_{k}(x)+\psi(k, j, x) \frac{\eta_{k}^{2}(x)}{\phi_{k, j}}\right),
\end{aligned}
$$

where, as a consequence of (3.13) and (3.14),

$$
|\psi(k, j, x)| \leq 1.2 \text {. }
$$

From (3.9) and (3.15) we have

$$
\begin{aligned}
& {\left[\int_{I_{k, j}} a(x)\left(\frac{d u}{d x}-\frac{d u_{k}}{d x}\right)^{2} d x\right]^{1 / 2} \geq \sqrt{\alpha}\left|u-u_{k}\right|_{H^{1}\left(I_{k, j}\right)}} \\
& \quad \geq \frac{\sqrt{\alpha}}{\phi_{k, j}^{2}}\left[\left(\int_{I_{k, j}} \eta_{k}^{2}(x) d x\right)^{1 / 2}-\frac{1}{\phi_{k, j}}\left(\int_{I_{k, j}} \psi^{2}(k, j, x) \eta_{k}^{4}(x) d x\right)^{1 / 2}\right] .
\end{aligned}
$$

Using (3.13), (3.14), and (3.16), we see that

$$
\frac{1}{\phi_{k, j}}\left(\int_{I_{k, j}} \psi^{2}(k, j, x) \eta_{k}^{4}(x) d x\right)^{1 / 2} \leq 0.14\left(\int_{I_{k, j}} \eta_{k}^{2}(x) d x\right)^{1 / 2} .
$$


Thus from (3.12b), (3.13), and (3.16) we have

$$
\left[\int_{I_{k, j}} a(x)\left(\frac{d u}{d x}-\frac{d u_{k}}{d x}\right)^{2} d x\right]^{1 / 2} \geq 0.6\left(\int_{I_{k, j}} \eta_{k}^{2}(x) d x\right)^{1 / 2}=0.6 h_{k}^{1 / 2}\left(\sum_{l=k+1}^{\infty} d_{l}^{2}\right)^{1 / 2}
$$

and hence

$$
\left\|u-u_{k}\right\|_{E} \geq 0.6\left(\sum_{l=k+1}^{\infty} d_{l}^{2}\right)^{1 / 2}, \text { for all } k .
$$

For $k=0,1, \ldots$ let $i$ be such that $l_{i} \leq k \leq l_{i+1}-1$. Then, using (3.7), we have

$$
\sum_{l=k+1}^{\infty} d_{l}^{2}=\sum_{m=i+1}^{\infty} d_{l_{m}}^{2}=\gamma^{2} \sum_{m=i}^{\infty} \chi_{l_{m}}^{2} \geq \gamma^{2} \chi_{l_{i}}^{2} \geq \gamma^{2} \chi_{k}^{2} .
$$

Combining this estimate with (3.18), we get

$$
\left\|u-u_{k}\right\|_{E} \geq 0.6 \gamma \chi_{k} \geq\left(3 \cdot 10^{-2}\right) \chi_{k}
$$

which is the first estimate in (3.1).

Remark 3. It follows from the definitions of $a(x)$, the mesh family $\left\{\Delta_{k}\right\}$, and the finite element approximation $u_{k}$ that

$$
u_{1}=\cdots=u_{l_{1}-1}, u_{l_{1}}=\cdots=u_{l_{2}-1}, \ldots, u_{l_{i}}=\cdots=u_{l_{(i+1)}-1}, \ldots
$$

By a similar argument we get an upper estimate. In fact, we get

$$
\begin{aligned}
& \left(\int_{I_{k, j}} a(x)\left(\frac{d u}{d x}-\frac{d u_{k}}{d x}\right)^{2} d x\right)^{1 / 2} \leq \sqrt{\beta}\left|u-u_{k}\right|_{H^{1}\left(I_{k, j}\right)} \\
& \quad \leq \frac{\sqrt{\beta}}{\phi_{k, j}^{2}}\left[\left(\int_{I_{k, j}} \eta_{k, j}^{2} d x\right)^{1 / 2}+\frac{1}{\phi_{k, j}}\left(\int_{I_{k, j}} \psi^{2}(k, j, x) \eta_{k}^{4}(x) d x\right)^{1 / 2}\right] \\
& \quad \leq 1.8\left(\int_{I_{k, j}} \eta_{k, j}^{2}(x) d x\right)^{1 / 2} \\
& \quad=1.8 h_{k}^{1 / 2}\left(\sum_{l=k+1}^{\infty} d_{l}^{2}\right)^{1 / 2} .
\end{aligned}
$$

Now, with $l_{i} \leq k \leq l_{(i+1)}-1$, using (3.5a), (3.5b), and (3.7), we have

$$
\sum_{l=k+1}^{\infty} d_{l}^{2}=\gamma^{2} \sum_{m=i}^{\infty} \chi_{l_{m}}^{2} \leq \gamma^{2} \chi_{l_{i}}^{2} \sum_{m=0}^{\infty} \gamma^{2 m}=\frac{\gamma^{2} \chi_{l_{i}}^{2}}{1-\gamma^{2}}<\frac{\chi_{l_{(i+1)}-1}^{2}}{1-\gamma^{2}} \leq \frac{\chi_{k}^{2}}{1-\gamma^{2}} .
$$

Combining this estimate with (3.21), we get

$$
\left\|u-u_{k}\right\|_{E} \leq \frac{1.8 \chi_{k}}{\sqrt{1-\gamma^{2}}} \leq 1.9 \chi_{k}, \text { for all } k
$$

which is the second estimate in (3.1).

Remark 4. It is possible to base Theorem 1 on a standard result in approximation theory. It is known [8], [16] that given any sequence $\left\{\chi_{k}\right\}$, there is a $u \in H_{0^{l}}^{1}(I)$ such that $\inf _{\phi \in \mathcal{V}_{k}}|u-\phi|_{H^{1}}=\chi_{k}$ for all $k$, i.e., there is a function $u$ with specified 
approximation properties. Now, we are interested in a solution $u$ that corresponds to a coefficient $a(x): u=u_{a}$. It immediate from (2.1) that $a(x)$ must be given by

$$
a(x)=\frac{1}{d u(x) / d x} .
$$

It is clear that $a(x)$, defined by (3.24), satisfies (2.2) if and only if $d u(x) / d x$ is bounded away from 0 and $\infty$. Now, it is not clear that the function $u$ constructed in [16] is in $L_{\infty}$, but with an alternate construction by P. Oswald [13], this is clear. Then by considering $\tilde{u}(x)=u(x)+c x$, for an appropriate value for $c$, we get a solution $\tilde{u}(x)$ that has specified approximations properties, and such that the corresponding $a(x)$ satisfies (2.1). In this way we obtain an alternate proof of Theorem 1.

We have chosen to prove Theorem 1 as we did, however, because it leads naturally to the proofs of Theorems 2 and 3 below. We thank Peter Oswald for pointing out the approximation theory result [8], [16] and the construction [13] mentioned above.

Remark 5. With $\chi_{k}=2^{-2 k}$, Theorem 1 shows that $\left\|u-u_{k}\right\|_{E}$ is of order $\chi_{k}=$ $2^{-2 k}=h_{k}^{2}$. This might seem to contradict the well known result that the highest possible rate of convergence with piecewise linear elements is $O(h)$, which is proved using the theory of $N$-widths (see, e.g., [14) and a saturation theorem (see, e.g., [9]). But the theory of $N$-widths is concerned with the worst possible case, and saturation theorems assume sufficient smoothness, so there is no contradiction.

\section{Analysis of the ERror at the nODAL POINTS}

We again let $u$ be the solution of (2.3) and let $u_{k}$ be the finite element approximation determined by $\Delta_{k}$. In this section we assess the error at the nodal points.

Theorem 2. Let $\left\{\chi_{k}\right\}_{k=0}^{\infty}$, with $\chi_{0}=1$, be a sequence of nonincreasing positive numbers converging to 0. Assume that the coefficient $a(x)$ is defined by (3.5a), (3.6), and (3.7). Then

$$
\left(1.5 \cdot 10^{-3}\right) \chi_{k}^{2} x_{k, j} \leq\left|\left(u-u_{k}\right)\left(x_{k, j}\right)\right| \leq 1.7 \chi_{k}^{2} x_{k, j}, j=1,2, \ldots, 2^{k}, \text { for all } k \text {. }
$$

Proof. We begin with a refinement of equation (3.15): For $x \in I_{k, j}$,

$$
\begin{aligned}
\frac{d u}{d x}-\frac{d u_{k}}{d x} & =\frac{-\eta_{k}(x)}{\phi_{k, j}^{2}\left(1+\frac{\eta_{k}(x)}{\phi_{k, j}}\right)} \\
& =\frac{1}{\phi_{k, j}^{2}}\left(-\eta_{k}(x)+\frac{\eta_{k}^{2}(x)}{\phi_{k, j}}-\psi(k, j, x) \frac{\eta_{k}^{3}(x)}{\phi_{k, j}^{2}}\right),
\end{aligned}
$$

where $\psi$ satisfies (3.16). Thus, using (3.12a), we have

$$
\int_{I_{k, j}}\left(\frac{d u}{d x}-\frac{d u_{k}}{d x}\right) d x=\frac{1}{\phi_{k, j}^{3}}\left[\int_{I_{k, j}} \eta_{k}^{2}(x) d x-\frac{\int_{I_{k, j}} \psi(k, j, x) \eta_{k}^{3}(x) d x}{\phi_{k, j}}\right] .
$$

Now, using (3.13), (3.14), and (3.16), we have

$$
\left|\frac{\int_{I_{k, j}} \psi(k, j, x) \eta_{k}^{3}(x) d x}{\phi_{k, j}}\right| \leq 0.14 \int_{I_{k, j}} \eta_{k}^{2}(x) d x .
$$


Thus, from (3.12b) and (3.14) we have

$$
\begin{aligned}
\int_{I_{k, j}}\left(\frac{d u}{d x}-\frac{d u_{k}}{d x}\right) d x & \geq 0.6 \int_{I_{k, j}} \eta_{k}^{2}(x) d x \\
& =0.6 h_{k} \sum_{l=k+1}^{\infty} d_{l}^{2}, \text { for all } k
\end{aligned}
$$

Hence

$$
\begin{aligned}
\left(u-u_{k}\right)\left(x_{k, 1}\right) & =\left(u-u_{k}\right)\left(x_{k, 0}\right)+\int_{x_{k, 0}}^{x_{k, 1}}\left(\frac{d u}{d x}-\frac{d u_{k}}{d x}\right) d x \\
& \geq 0.6\left(\sum_{l=k+1}^{\infty} d_{l}^{2}\right) h_{k} \\
& =0.6\left(\sum_{l=k+1}^{\infty} d_{l}^{2}\right) x_{k, 1}, \\
\left(u-u_{k}\right)\left(x_{k, 2}\right) & =\left(u-u_{k}\right)\left(x_{k, 1}\right)+\int_{x_{k, 1}}^{x_{k, 2}}\left(\frac{d u}{d x}-\frac{d u_{k}}{d x}\right) d x \\
& \geq 0.6\left(\sum_{l=k+1}^{\infty} d_{l}^{2}\right)\left(x_{k, 1}+h_{k}\right) \\
& =0.6\left(\sum_{l=k+1}^{\infty} d_{l}^{2}\right) x_{k, 2},
\end{aligned}
$$

and, in general,

$$
\left(u-u_{k}\right)\left(x_{k, j}\right) \geq 0.6\left(\sum_{l=k+1}^{\infty} d_{l}^{2}\right) x_{k, j}, \quad j=1, \ldots, 2^{k}, \text { for all } k .
$$

Combining (3.19) and (4.5), we get

$$
\left(u-u_{k}\right)\left(x_{k, j}\right) \geq 0.6 \gamma^{2} \chi_{k}^{2} x_{k, j}=\left(1.5 \cdot 10^{-3}\right) \chi_{k}^{2} x_{k, j},
$$

which is the first estimate in (4.1).

A similar argument yields

$$
\left(u-u_{k}\right)\left(x_{k, j}\right) \leq 1.6\left(\sum_{l=k+1}^{\infty} d_{l}^{2}\right) x_{k, j}
$$

Combining this estimate with (3.22) yields

$$
\left(u-u_{k}\right)\left(x_{k, j}\right) \leq 1.7 \chi_{k}^{2} x_{k, j},
$$

which is the second estimate in (4.1).

Remark 6. From the usual finite element error analysis, we know that the nodal point errors are 0 if $a(x)=$ constant, and are $O\left(h^{2}\right)$ if $a(x)$ is smooth. We note that neither of these results applies to our example. Note that we obtained arbitrarily low rates of convergence by appropriately selecting the sequence $\chi_{k}$; the corresponding coefficient $a(x)$ is nearly constant (cf. (3.9)), but is not smooth. 
5. ANALYSIS OF THE ERROR IN THE $L_{2}$-NORM

The usual duality argument [1], [11], [12] shows that

$$
\left\|u-u_{k}\right\|_{L_{2}(I)} \leq \eta(h)\left\|u-u_{k}\right\|_{E},
$$

where

$$
\eta(h)=\sup _{\phi \in L_{2}(I)} \frac{\inf _{\tau \in \mathcal{V}_{k}}\left\|w_{\phi}-\tau\right\|_{E}}{\|\phi\|_{L_{2}(I)}},
$$

$w_{\phi}$ being the solution of

$$
\left\{\begin{array}{l}
-\frac{d}{d x}\left(a(x) \frac{d w_{\phi}}{d x}\right)=\phi, \quad 0<x<1, \\
w_{\phi}(0)=0, \quad a w_{\phi}^{\prime}(1)=0 .
\end{array}\right.
$$

If $a(x)$ is smooth, specifically if $a(x) \in C^{1}[0,1]$, then $\eta(h) \leq C h$, and thus

$$
\left\|u-u_{k}\right\|_{L_{2}(I)} \leq C h\left\|u-u_{k}\right\|_{E}
$$

and

$$
\left\|u-u_{k}\right\|_{L_{2}(I)} \leq C\left\|u-u_{k}\right\|_{E}^{2} .
$$

Estimate (5.3) follows from (5.2) and the estimate $\left\|u-u_{k}\right\|_{E} \geq C h$, which is valid under a mild hypothesis on $u$ (in addition to smoothness) [6]. But if $a(x)$ is rough, if we are assuming it is merely measurable, then, although $\lim _{h \rightarrow 0} \eta(h)=0$, no estimate of the form $\eta(h) \leq C h^{\rho}$ with $\rho>0$ may hold. In this situation, we may know [5], 15] only that

$$
\left\|u-u_{k}\right\|_{L_{2}(I)}=o\left(\left\|u-u_{k}\right\|_{E}\right) .
$$

In this section we derive estimates on the $L_{2}(I)$-error. Following their derivation, we discuss their relation with $L_{2}$-estimates derived via duality. We will also state estimates on the $L_{\infty}$-error, and discuss their relation with superconvergence at the nodes.

Theorem 3. Let $\left\{\chi_{k}\right\}_{k=0}^{\infty}$, with $\chi_{0}=1$, be a sequence of nonincreasing positive numbers converging to 0 . Assume that the coefficient $a(x)$ is defined by $(3.5 a)$ and (3.6), and (3.7). Then

$$
\begin{aligned}
\max \left\{\left(1.4 \cdot 10^{-3}\right) \chi_{l_{i}-1}\right. & \left.h_{l_{i}-1},\left(0.53 \cdot 10^{-3}\right) \chi_{l_{i}-1}^{2}-0.36 \chi_{l_{i}-1} h_{l_{i}-1}\right\} \\
& \leq\left\|u-u_{l_{i}-1}\right\|_{L_{2}(I)} \\
& \leq 1.7 \chi_{l_{i}-1}^{2}+0.5 \chi_{l_{i}-1} h_{l_{i}-1}, \text { for all } i \geq 2 .
\end{aligned}
$$

Proof. Using (4.2) with $k=l_{i}-1$, for $x \in I_{l_{i}-1, j}$ we have

$$
\begin{aligned}
\left(u-u_{l_{i}-1}\right)(x)= & \left(u-u_{l_{i}-1}\right)\left(x_{l_{i}-1 . j-1}\right)+\int_{x_{l_{i}-1 . j-1}}^{x}\left[u^{\prime}(t)-u_{l_{i}-1}^{\prime}(t)\right] d t \\
= & \left(u-u_{l_{i}-1}\right)\left(x_{l_{i}-1 . j-1}\right)-\frac{1}{\phi_{l_{i}-1, j}^{2}} \int_{x_{l_{i}-1, j-1}}^{x} \eta_{l_{i}-1}(t) d t \\
& +\frac{1}{\phi_{l_{i}-1, j}^{3}} \int_{x_{l_{i}-1, j-1}}^{x} \eta_{l_{i}-1}^{2}(t) d t \\
& -\frac{1}{\phi_{l_{i}-1, j}^{4}} \int_{x_{l_{i}-1, j-1}}^{x} \psi\left(l_{i}-1, j, t\right) \eta_{l_{i}-1}^{3}(t) d t .
\end{aligned}
$$


From (5.6) and the definition of $\eta_{l_{i}-1}(x)$ (equation (3.10)) we get

$$
\begin{aligned}
& \left\|u-u_{l_{i}-1}\right\|_{L_{2}\left(I_{l_{i}-1, j}\right)} \\
& =\|\left(u-u_{l_{i}-1}\right)\left(x_{l_{i}-1, j-1}\right)-\frac{d_{l_{i}}}{\phi_{l_{i}-1, j}} \int_{x_{l_{i}-1, j-1}}^{x} g_{l_{i}}(t) d t \\
& \quad-\frac{1}{\phi_{l_{i}-1, j}^{2}} \sum_{m=i+1}^{\infty} d_{l_{m}} \int_{x_{l_{i}-1, j-1}}^{x} g_{l_{m}}(t) d t \\
& \quad+\frac{1}{\phi_{l_{i}-1, j}^{3}} \int_{x_{l_{i}-1, j-1}}^{x} \eta_{l_{i}-1}^{2}(t) d t \\
& \quad-\frac{1}{\phi_{l_{i}-1, j}^{4}} \int_{x_{l_{i}-1, j-1}}^{x} \psi\left(l_{i}-1, j, t\right) \eta_{l_{i}-1}^{3}(t) d t \|_{L_{2}\left(I_{l_{i}-1, j}\right)} \\
& \geq \frac{d_{l_{i}}}{\phi_{l_{i}-1, j}^{2}}\left\|\int_{x_{l_{i}-1, j-1}}^{x} g_{l_{i}}(t) d t-\frac{\phi_{l_{i}-1, j}^{2}}{d_{l_{i}}}\left(u-u_{l_{i}-1}\right)\left(x_{l_{i}-1, j-1}\right)\right\|_{L_{2}\left(I_{l_{i}-1, j}\right)} \\
& \quad-\frac{1}{\phi_{l_{i}-1, j}^{2}} \sum_{m=i+1}^{\infty} d_{l_{m}}\left\|\int_{x_{l_{i}-1, j-1}}^{x} g_{l_{m}}(t) d t\right\|_{L_{2}\left(I_{l_{i}-1, j}\right)} \\
& \quad-\frac{1}{\phi_{l_{i}-1, j}^{3}}\left\|\int_{x_{l_{i}-1, j-1}}^{x} \eta_{l_{i}-1}^{2}(t) d t\right\|_{L_{2}\left(I_{l_{i}-1, j}\right)} \\
& \quad-\frac{1}{\phi_{l_{i}-1, j}^{4}}\left\|\int_{x_{l_{i}-1, j-1}}^{x} \psi\left(l_{i}-1, j, t\right) \eta_{l_{i}-1}^{3}(t) d t\right\|_{L_{2}\left(I_{l_{i}-1, j}\right)} \\
& =A
\end{aligned}
$$

We will estimate each of the terms on the right side of (5.7) in turn.

Let

$$
G_{l_{m}}(x)=\int_{x_{l_{i}-1, j-1}}^{x} g_{l_{m}}(t) d t .
$$

The graph of $G_{l_{i}}(x)$ on the interval $I_{l_{i}-1, j}$ is shown in Figure 1.

A direct calculation shows that

$$
\left\|G_{l_{i}}\right\|_{L_{2}\left(I_{l_{i}-1, j}\right)}=\frac{h_{l_{i}-1}^{3 / 2}}{2 \sqrt{3}}
$$

Now

$$
\int_{I_{l_{i}-1}}\left|G_{l_{i}}(x)-c\right|^{2} d x
$$

is minimized by

$$
c=\bar{c}=\frac{\int_{I_{l_{i}-1, j}} G_{l_{i}}(x) d x}{h_{l_{i}-1}} .
$$




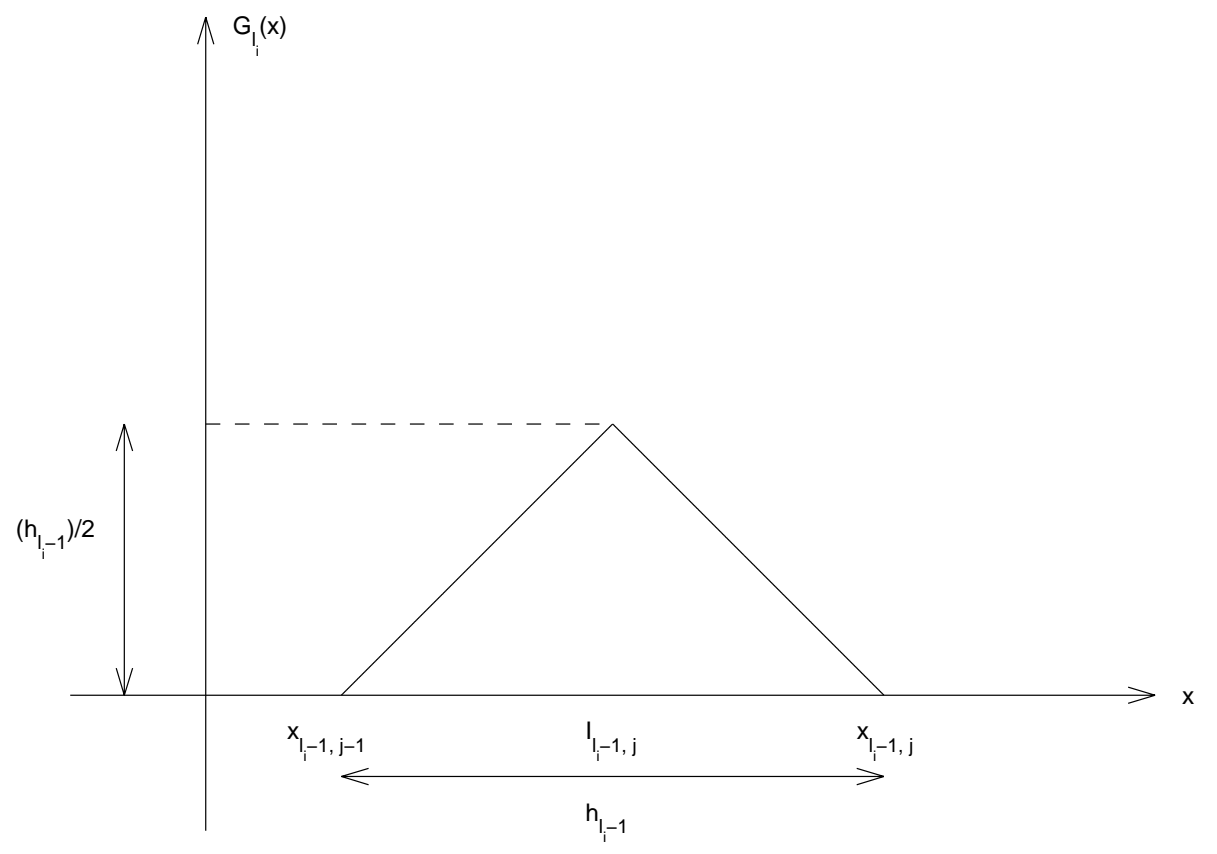

FiguRE 1.

Thus, another direct calculation yields

$$
\begin{aligned}
& {\left[\int_{I_{l_{i}-1, j}}\left|G_{l_{i}}(x)-\frac{\phi_{k, j}^{2}}{d_{l_{i}}}\left(u-u_{l_{i}-1}\right)\left(x_{l_{i}-1, j-1}\right)\right|^{2} d x\right]^{1 / 2}} \\
& \quad \geq \sqrt{\int_{I_{l_{i}-1, j}}\left|G_{l_{i}}(x)-\bar{c}\right|^{2} d x} \\
& \quad=\frac{h_{l_{i}-1}^{3 / 2}}{4 \sqrt{3}}
\end{aligned}
$$

Hence, using (3.7) and (3.13), we have

$$
\begin{aligned}
A & =\frac{d_{l_{i}}}{\phi_{k, j}^{2}}\left\|\int_{x_{l_{i}-1, j-1}}^{x} g_{l_{i}}(t) d t-\frac{\phi_{k, j}^{2}}{d_{l_{i}}}\left(u-u_{l_{i}-1}\right)\left(x_{l_{i}-1, j-1}\right)\right\|_{L_{2}\left(I_{l_{i}-1, j}\right)} \\
& =\frac{\gamma \chi_{l_{i-1}}}{\phi_{k, j}^{2}}\left[\int_{I_{l_{i}-1, j}}\left|G_{l_{i}}(x)-\frac{\phi_{k, j}^{2}}{d_{l_{i}}}\left(u-u_{l_{i}-1}\right)\left(x_{l_{i}-1, j-1}\right)\right|^{2} d x\right]^{1 / 2} \\
& \geq\left(5.9 \cdot 10^{-3}\right) h_{l_{i}-1}^{3 / 2} \chi_{l_{i-1}} .
\end{aligned}
$$




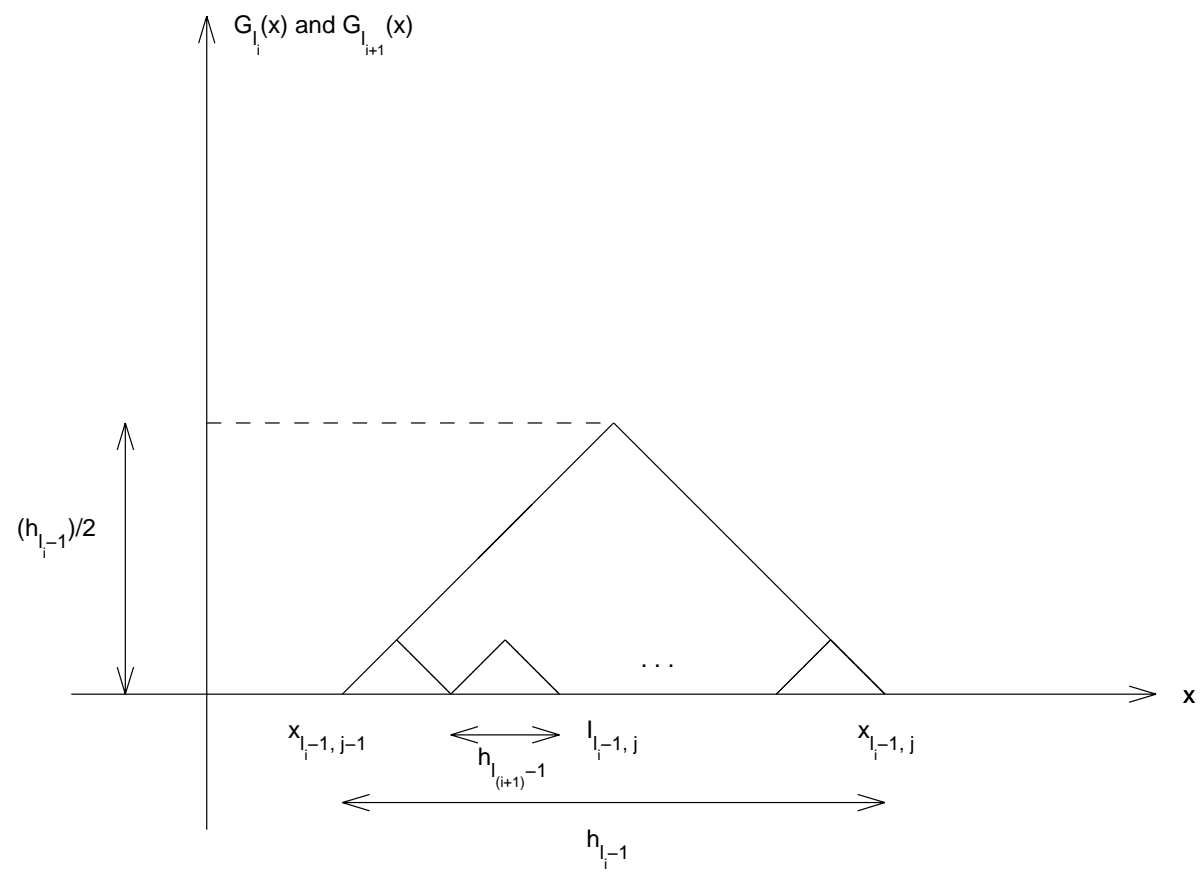

FIGURE 2.

The graphs of $G_{l_{i}}(x)$ and $G_{l_{i+1}}(x)$ on the interval $I_{l_{i}-1, j}$ are shown in Figure 2. Another direct calculation shows that

$$
\begin{aligned}
\left\|G_{l_{i+1}}\right\|_{L_{2}\left(I_{l_{i}-1, j}\right)}^{2} & =\frac{h_{l_{i+1}-1}^{3}}{12} \frac{h_{l_{i}-1}}{h_{l_{i+1}-1}} \\
& =\left\|G_{l_{i}}\right\|_{L_{2}\left(I_{l_{i}-1, j}\right)}^{2}\left(\frac{h_{l_{i+1}-1}}{h_{l_{i}-1}}\right)^{2} \\
& =\left\|G_{l_{i}}\right\|_{L_{2}\left(I_{l_{i}-1, j}\right)}^{2}\left(\frac{1}{2^{\left(l_{i+1}-l_{i}\right)}}\right)^{2} \\
& \leq \frac{1}{4}\left\|G_{l_{i}}\right\|_{L_{2}\left(I_{l_{i}-1}, j\right)}^{2} ;
\end{aligned}
$$

and, in general,

$$
\left\|G_{l_{i+s}}\right\|_{L_{2}\left(I_{l_{i}-1, j}\right)} \leq \frac{1}{2^{s}}\left\|G_{l_{i}}\right\|_{L_{2}\left(I_{l_{i}-1}, j\right)} .
$$


So, using (3.5a), (3.7), and (5.8), we have

$$
\begin{aligned}
\sum_{m=i+1}^{\infty} d_{l_{m}}\left\|\int_{x_{l_{i}-1, j-1}}^{x} g_{l_{m}}(t) d t\right\|_{L_{2}\left(I_{l_{i}-1, j}\right)} & =\sum_{m=i+1}^{\infty} d_{l_{m}}\left\|G_{l_{m}}\right\|_{L_{2}\left(I_{l_{i}-1, j}\right)} \\
& \leq\left\|G_{l_{i}}\right\|_{L_{2}\left(I_{l_{i}-1, j}\right)} \sum_{m=i+1}^{\infty} \frac{d_{l_{m}}}{2^{m-i}} \\
& =\left\|G_{l_{i}}\right\|_{L_{2}\left(I_{l_{i}-1, j}\right)} \sum_{m=i+1}^{\infty} \frac{\gamma \chi_{l_{m-1}}}{2^{m-i}} \\
& \leq\left\|G_{l_{i}}\right\|_{L_{2}\left(I_{l_{i}-1, j}\right)} \frac{\gamma^{2} \chi_{l_{i-1}}}{2} \sum_{m=0}^{\infty}\left(\frac{\gamma}{2}\right)^{m} \\
& =\frac{\gamma^{2}}{2 \sqrt{3}(2-\gamma)} h_{l_{i}-1}^{3 / 2} \chi_{l_{i-1}} .
\end{aligned}
$$

Hence

$$
B \leq\left(4.6 \cdot 10^{-4}\right) h_{l_{i}-1}^{3 / 2} \chi_{l_{i-1}} .
$$

In a similar way we get

$$
B^{\prime}=\frac{1}{\phi_{l_{i}-1, j}^{2}} \sum_{m=i}^{\infty} d_{l_{m}}\left\|\int_{x_{l_{i}-1, j-1}}^{x} g_{l_{m}}(t) d t\right\|_{L_{2}\left(I_{l_{i}-1, j}\right)} \leq\left(2 \cdot 10^{-2}\right) h_{l_{i}-1}^{3 / 2} \chi_{l_{i-1}} ;
$$

this estimate will be used later.

Using (3.5a), (3.7), and (3.12b), we have

$$
\begin{aligned}
\int_{I_{l_{i}-1, j}}\left|\int_{x_{l_{i}-1, j-1}}^{x} \eta_{l_{i}-1}^{2}(t) d t\right|^{2} d x & \leq \int_{I_{l_{i}-1, j}}\left|\int_{I_{l_{i}-1, j}} \eta_{l_{i}-1}^{2}(t) d t\right|^{2} d x \\
& \leq\left(\int_{I_{l_{i}-1, j}} \eta_{l_{i}-1}^{2}(t) d t\right)^{2} h_{l_{i}-1} \\
& =h_{l_{i}-1}^{3}\left(\sum_{m=i}^{\infty} d_{l_{m}}^{2}\right)^{2} \\
& =h_{l_{i}-1}^{3}\left(\sum_{m=i}^{\infty} \gamma^{2} \chi_{l_{m-1}}^{2}\right)^{2} \\
& \leq h_{l_{i}-1}^{3} \gamma^{4} \chi_{l_{i-1}}^{4}\left(\sum_{m=i}^{\infty} \gamma^{2(m-i)}\right)^{2} \\
& =\left(\frac{\gamma^{2}}{1-\gamma^{2}}\right)^{2} h_{l_{i}-1}^{3} \chi_{l_{i-1}}^{4} \\
& =\left(\frac{1}{399}\right)^{2} h_{l_{i}-1}^{3} \chi_{l_{i-1}}^{4}
\end{aligned}
$$

Hence

$$
C \leq\left(3.5 \cdot 10^{-3}\right) h_{l_{i-1}}^{3 / 2} \chi_{l_{i-1}}
$$


Using (3.14), (3.16), and (5.11), we get

$$
\begin{aligned}
\int_{I_{l_{i}-1, j-1}} \mid \int_{x_{l_{i}-1, j-1}}^{x} & \left.\psi\left(l_{i}-1, j, t\right) \eta_{l_{i}-1}^{3}(t) d t\right|^{2} d x \\
\leq & (0.12)^{2}\left|\int_{I_{l_{i}-1, j-i}} \int_{x_{l_{i}-1, j-1}}^{x} \eta_{l_{i}-1}^{2}(t) d t\right|^{2} d x \\
& \leq\left(\frac{0.12}{399}\right)^{2} h_{l_{i}-1}^{3} \chi_{l_{i}-1}^{4} .
\end{aligned}
$$

Hence

$$
D \leq\left(4.6 \cdot 10^{-4}\right) h_{l_{i}-1}^{3 / 2} \chi_{l_{i-1}}
$$

Finally, combining (5.7), (5.9), (5.10a), (5.12), and (5.13), we get

$$
\left\|u-u_{l_{i}-1}\right\|_{L_{2}\left(I_{l_{i}-1, j}\right)} \geq\left(1.4 \cdot 10^{-3}\right) h_{l_{i}-1}^{3 / 2} \chi_{l_{i}-1},
$$

where in the last inequality we have used the fact that $l_{i-1} \leq l_{i}-1$, which implies $\chi_{l_{i-1}} \geq \chi_{l_{i}-1}$. It follows immediately from (5.14) that

$$
\left\|u-u_{l_{i}-1}\right\|_{L_{2}(I)} \geq\left(1.4 \cdot 10^{-3}\right) h_{l_{i}-1} \chi_{l_{i}-1} .
$$

We now prove another lower bound. From (5.6) we have

$$
\begin{aligned}
& \left\|u-u_{l_{i}-1}\right\|_{L_{2}\left(I_{l_{i}-1, j}\right)} \\
& \geq\left\|\left(u-u_{l_{i}-1}\right)\left(x_{l_{i}-1, j-1}\right)\right\|_{L_{2}\left(I_{l_{i}-1, j}\right)} \\
& -\| \frac{1}{\phi_{l_{i}-1, j}^{2}} \sum_{m=i}^{\infty} d_{l_{m}} \int_{x_{l_{i}-1, j-1}}^{x} g_{l_{m}}(t) d t \\
& \quad-\frac{1}{\phi_{l_{i}-1, j}^{3}} \int_{x_{l_{i}-1, j-1}}^{x} \eta_{l_{i}-1}^{2}(t) d t \\
& \quad+\frac{1}{\phi_{l_{i}-1, j}^{4}} \int_{x_{l_{i}-1, j-1}}^{x} \psi\left(l_{i}-1, j, t\right) \eta_{l_{i}-1}^{3}(t) d t \|_{L_{2}\left(I_{l_{i}-1, j}\right)} \\
& \geq\left|\left(u-u_{l_{i}-1}\right)\left(x_{l_{i}-1, j-1}\right)\right| h_{l_{i}-1}^{1 / 2} \\
& \quad-\frac{1}{\phi_{l_{i}-1, j}^{2}} \sum_{m=i}^{\infty} d_{l_{m}}\left\|\int_{x_{l_{i}-1, j-1}}^{x} g_{l_{m}}(t) d t\right\|_{L_{2}\left(I_{l_{i}-1, j}\right)} \\
& \quad-\frac{1}{\phi_{l_{i}-1, j}^{3}}\left\|\int_{x_{l_{i}-1, j-1}}^{x} \eta_{l_{i}-1}^{2}(t) d t\right\|_{L_{2}\left(I_{l_{i}-1, j}\right)} \\
& \quad-\frac{1}{\phi_{l_{i}-1, j}^{4}}\left\|\int_{x_{l_{i}-1, j-1}}^{x} \psi\left(l_{i}-1, j, t\right) \eta_{l_{i}-1}^{3}(t) d t\right\|_{L_{2}\left(I_{l_{i}-1, j}\right)} \\
& =A^{\prime}-B^{\prime}-C-D .
\end{aligned}
$$

From (4.6) with $k=l_{i}-1$ we have

$$
A^{\prime} \geq\left(1.5 \cdot 10^{-3}\right) \chi_{l_{i}-1}^{2} x_{l_{i}-1, j-1} h_{l_{i}-1}^{1 / 2} .
$$

From (3.5b), (5.10b), (5.12), and (5.13) we have

$$
B^{\prime}+C+D \leq\left(2.4 \cdot 10^{-2}\right) h_{l_{i}-1}^{3 / 2} \chi_{l_{i-1}}<\left(2.4 \cdot 10^{-2}\right) h_{l_{i}-1}^{3 / 2} \gamma^{-1} \chi_{l_{i}-1} \leq 0.5 h_{l_{i}-1}^{3 / 2} \chi_{l_{i}-1} .
$$


Combining (5.16), (5.17), and (5.18), we have

$$
\left\|u-u_{l_{i}-1}\right\|_{L_{2}\left(I_{l_{i}-1, j}\right)} \geq\left[\left(1.5 \cdot 10^{-3}\right) x_{l_{i}-1, j-1} \chi_{l_{i}-1}^{2}-0.5 h_{l_{i}-1} \chi_{l_{i}-1}\right] h_{l_{i}-1}^{1 / 2} .
$$

Now consider intervals $I_{l_{i}-1, j}$ such that $x_{l_{i}-1, j-1} \geq 1 / 2$, i.e., consider $j$ 's satisfying $2^{l_{i}-2}+1 \leq j \leq 2^{l_{i}-1}$. There are $2^{l_{i}-2}$ such $j$ 's if $i \geq 2$, and for each of them we have

$$
\left\|u-u_{l_{i}-1}\right\|_{L_{2}\left(I_{l_{i}-1, j}\right)} \geq\left[\left(1.5 \cdot 10^{-3}\right) \frac{1}{2} \chi_{l_{i}-1}^{2}-0.5 h_{l_{i}-1} \chi_{l_{i}-1}\right] h_{l_{i}-1}^{1 / 2} .
$$

Suppose $\left(0.75 \cdot 10^{-3}\right) \chi_{l_{i}-1}-0.5 h_{l_{i}-1}>0$. Then

$$
\begin{aligned}
\left\|u-u_{l_{i}-1}\right\|_{L_{2}(I)}^{2} & \geq\left\|u-u_{l_{i}-1}\right\|_{L_{2}(1 / 2,0)}^{2} \\
& =\sum_{2^{l_{i}-2}+1 \leq j \leq 2^{l_{i}-1}}\left\|u-u_{l_{i}-1}\right\|_{L_{2}\left(I_{l_{i}-1, j}\right)}^{2} \\
& \geq\left[\left(0.75 \cdot 10^{-3}\right) \chi_{l_{i}-1}^{2}-0.5 h_{l_{i}-1} \chi_{l_{i}-1}\right]^{2} h_{l_{i}-1} 2^{l_{i}-2} \\
& =\frac{1}{2}\left[\left(0.75 \cdot 10^{-3}\right) \chi_{l_{i}-1}^{2}-0.5 h_{l_{i}-1} \chi_{l_{i}-1}\right]^{2}, \text { for } i \geq 2 .
\end{aligned}
$$

Hence

$$
\left\|u-u_{l_{i}-1}\right\|_{L_{2}(I)} \geq\left(0.53 \cdot 10^{-3}\right) \chi_{l_{i}-1}^{2}-0.36 h_{l_{i}-1} \chi_{l_{i}-1} .
$$

We easily see that $(5.19)$ is valid without the assumption that $\left(0.75 \cdot 10^{-3}\right) \chi_{l_{i}-1}-$ $0.5 h_{l_{i}-1}>0$. Estimates (5.15) and (5.19) prove the first estimate in (5.5).

Now we prove the second estimate in (5.5). From (5.6) we have

$$
\left\|u-u_{l_{i}-1}\right\|_{L_{2}\left(I_{l_{i}-1}, j\right)} \leq A^{\prime}+B^{\prime}+C+D \text {. }
$$

Estimate (4.7) with $k=l_{i}-1$ yields

$$
A^{\prime} \leq 1.7 \chi_{l_{i}-1}^{2} h_{l_{i}-1}^{1 / 2} .
$$

Combining (5.18), (5.20), and (5.21) yields

$$
\left\|u-u_{l_{i}-1}\right\|_{L_{2}\left(I_{l_{i}-1, j}\right)} \leq\left[1.7 \chi_{l_{i}-1}^{2}+0.5 \chi_{l_{i}-1} h_{l_{i}-1}\right] h_{l_{i}-1}^{1 / 2} .
$$

Thus

$$
\left\|u-u_{l_{i}-1}\right\|_{L_{2}(I)} \leq 1.7 \chi_{l_{i}-1}^{2}+0.5 \chi_{l_{i}-1} h_{l_{i}-1},
$$

which is the second estimate in (5.5).

Remark 7. It is informative to consider the following two cases:

Case 1 . The sequence $\chi_{k}$ converges rapidly to 0 ;

Case 2. The sequence $\chi_{k}$ converges slowly to 0

Suppose we are in Case 1 . Then the $l_{i}$ 's are not so large (we could, e.g., have $\left.l_{i}=i\right)$, and $\chi_{l_{i}-1} \ll h_{l_{i}-1}$. Thus from (5.5) we see that $\left\|u-u_{l_{i}-1}\right\|_{L_{2}(I)}$ is of order $h_{l_{i}-1} \chi_{l_{i}-1}$. Combining this result with (3.1) shows that

$$
\left\|u-u_{l_{i}-1}\right\|_{L_{2}(I)} \text { is of order } h_{l_{i}-1}\left\|u-u_{l_{i}-1}\right\|_{E} .
$$

Suppose next we are in Case 2, which we are mainly interested in. Then the $l_{i}$ 's are very large, so $\chi_{l_{i}-1} \gg h_{l_{i}-1}$. Thus (5.5) shows that $\left\|u-u_{l_{i}-1}\right\|_{L_{2}(I)}$ is of order $\chi_{l_{i}-1}^{2}$ Combining this result with (3.1) shows that

$$
\left\|u-u_{l_{i}-1}\right\|_{L_{2}(I)} \text { is of order }\left\|u-u_{l_{i}-1}\right\|_{E}^{2} \text {. }
$$


We can relate these results to estimates (5.2), (5.3), and (5.6). We see that in Case 1, an estimate of type (5.2) holds, but not (5.3). In Case 2, an estimate of type (5.3) holds, but not (5.2). The duality argument used to prove (5.2) and (5.3) is, of course, not valid for our example, since it is not smooth. We have given direct alternate proofs of (5.22) and (5.23).

Although (5.3) is proved only for smooth problems, if it is formally considered for a rough problem, it is similar to (5.4) in that the upper bound is a quantity that goes to zero-possibly very slowly-times $\left\|u-u_{k}\right\|_{E}$. In this sense (5.23) is compatible with (5.4).

Finally we note that although our example is rough in both case, Case 1 does have the following "smoothness": The series defining $a(x)$ is "less lacunary", so $a(x)$, and hence $u(x)$, is "smooth".

Remark 8. With an analysis similar that used in the proof of Theorem 3, one can obtain bounds for the $L_{\infty}$-error. These bounds have the same form as those in (5.5). Specifically, one can show that

$$
\begin{aligned}
\max \left\{C_{1} \chi_{l_{i}-1} h_{l_{i}-1}, C_{2} \chi_{l_{i}-1}^{2}-C_{3} \chi_{l_{i}-1} h_{l_{i}-1}\right\} & \leq\left\|u-u_{l_{i}-1}\right\|_{L_{\infty}(I)} \\
& \leq C_{4} \chi_{l_{i}-1}^{2}+C_{5} \chi_{l_{i}-1} h_{l_{i}-1} .
\end{aligned}
$$

In Case 1 (see Remark 7), (4.1) and (5.24) show that the $L_{\infty}$-error is of order $h_{l_{i}-1} \chi_{l_{i}-1}$, and that the nodal point errors are of the higher order $\chi_{l_{i}-1}^{2}$, establishing superconvergence at the nodes. This is the error-behavior we expect with a smooth problem. In Case 2, the nodal point errors and the $L_{\infty}$-error are of order $\chi_{l_{i}-1}^{2}$, showing that there is no superconvergence at the nodes. This is the error-behavior we expect with a rough problem.

Remark 9. The same coefficient $a(x)$ is used in all three theorems. To be precise, with $\gamma=1 / 20$ and with $a(x)$ defined in (3.5a), (3.6), and (3.7), estimates (3.1), (4.1), and (5.5) for the energy-norm error, the nodal point errors, and the $L_{2}$-error, respectively, hold simultaneously.

\section{Adaptivity}

So far we have worked with uniform meshes. Consider now a family of meshes with nodes of the form $j 2^{-k}$, with $j$ taking on a sequence of $N+1$ values between 0 and $2^{k}$, inclusive. Such mesh families are often constructed by adaptive procedures, and in most practical situations these adaptive procedures produce mesh families and associated approximate solutions whose rate of convergence, measured in the energy norm, is $O(1 / N)$. Suppose we use the following, typical, adaptive procedure. Starting from a uniform mesh, we consider the energy norm error on each subinterval of the mesh. We then refine, by dividing in two equal parts, each subinterval whose error is greater than or equal to $\delta \times$ (maximum subinterval error), where $0<\delta<1$ is a specified parameter. Following this refinement, we repeat the process. The resulting mesh will in general depend on the solution $u(x)$, and thus on the coefficient $a(x)$. We denote the meshes by $\Delta_{N}^{a}$. This approach is based on the equilibrium principle, which tries to make the errors in the elements approximately equal. This principle is used in all adaptive approaches. For an analysis of this and similar approaches, we refer to [4], [7].

We examine this adaptive process for our boundary value problem, (2.1), with the coefficient $a(x)$ defined by (3.5a), (3.6), and (3.7). We claim that if $\delta<\frac{1}{2}$, and 
we start with the uniform mesh $\Delta_{0}=\{0,1\}$, then the adaptive procedure produces a uniform mesh family. To prove this, suppose that at some stage we have the uniform mesh $\Delta_{k}=\left\{0,2^{-k}, \cdots, 1\right\}$. It follows from (2.4), (3.3), (3.9), (3.10), and (3.13) that

$$
\begin{aligned}
\int_{I_{k, j}} a\left|\left(u-u_{k}\right)^{\prime}\right|^{2} d x & =\int_{I_{k}, j} \frac{\left|a_{k, j}-a(x)\right|^{2}}{a(x) a_{k, j}^{2}} d x \\
& \leq(0.9)^{-3} \int_{I_{k, j}}\left|a_{k, j}-a(x)\right|^{2} d x \\
& =(0.9)^{-3} \int_{I_{k, j}}\left|\phi_{k}(x)-a(x)\right|^{2} d x \\
& =(0.9)^{-3} \int_{I_{k, j}}\left|\eta_{k}(x)\right|^{2} d x
\end{aligned}
$$

and

$$
\begin{aligned}
\int_{I_{k, i}} a\left|\left(u-u_{k}\right)^{\prime}\right|^{2} d x & \geq(1.1)^{-3} \int_{I_{k, i}}\left|a_{k, i}-a(x)\right|^{2} d x \\
& =(1.1)^{-3} \int_{I_{k, i}}\left|\eta_{k}(x)\right|^{2} d x .
\end{aligned}
$$

Now, it follows from $(3.12 \mathrm{~b})$ that

$$
\int_{I_{k}, j}\left|\eta_{k}(x)\right|^{2} d x=\int_{I_{k, i}}\left|\eta_{k}(x)\right|^{2} d x
$$

Hence,

$$
\int_{I_{k, j}} a\left|\left(u-u_{k}\right)^{\prime}\right|^{2} d x \leq\left(\frac{1.1}{0.9}\right)^{3} \int_{I_{k, i}} a\left|\left(u-u_{k}\right)^{\prime}\right|^{2} d x \leq 2 \int_{I_{k, i}} a\left|\left(u-u_{k}\right)^{\prime}\right|^{2} d x
$$

for any two subintervals $I_{k, j}$ and $I_{k, i}$ of $\Delta_{k}$. Thus, if $\delta<\frac{1}{2}$, every subinterval is subdivided, and the refined mesh is $\Delta_{k+1}$. So, starting with $\Delta_{0}$, the adaptive procedure produces $\Delta_{1}, \Delta_{2}, \ldots$.

Since the adaptive procedure will produce only uniform meshes, we see that the error in the energy norm, the nodal point errors, and the error in $L_{2}$ are as indicated in Theorems 1, 2 and 3.

\section{Conclusions}

a) We have shown that there are problems whose finite element approximations converge arbitrarily slowly, and that adaptivity cannot improve this situation. Specifically, if $\chi_{k}$ converges to 0 , with $\chi_{0}=1$, then there is a problem such that

- the energy norm error is of order $\chi_{k}$;

- the nodal point errors are of order $\chi_{k}^{2}$; and

- $\left\|u-u_{l_{i}-1}\right\|_{L_{2}(I)}$ is of order $h_{l_{i}-1} \chi_{l_{i}-1}$ if $\chi_{k}$ converges rapidly to 0 , and is of order $\chi_{l_{i}-1}^{2}$ if $\chi_{k}$ converges slowly.

Adaptivity does not improve the convergence. 
b) The cases $\chi_{k} \rightarrow 0$ rapidly (Case 1 ) and $\chi_{k} \rightarrow 0$ slowly (Case 2) need to be distinguished; they lead to different behaviors. The relation between the $L_{2}(I)$-error and the energy norm error is compatible with the smooth problem estimate,

$$
\left\|u-u_{k}\right\|_{L_{2}(I)} \leq h_{k}\left\|u-u_{k}\right\|_{E}
$$

in Case 1, and it is compatible with the smooth problem estimate

in Case 2.

$$
\left\|u-u_{k}\right\|_{L_{2}(I)} \leq C\left\|u-u_{k}\right\|_{E}^{2}
$$

c) We have shown that we have superconvergence at the nodes in Case 1, but not in Case 2.

\section{REFERENCES}

1. A. Aubin, Behavior of the error of the approximate solution of boundary value problems for linear elliptic operators by Galerkin's and finite difference methods, Ann. Scoula Norm. Sup. Pisa 21 (1967), 599-637. MR 38:1391

2. I. Babuška G. Caloz, and J. Osborn, Special finite element methods for a class of second order boundary value problems with rough coefficients, SIAM J. Numer. Anal. 31 (1994), 945-981. MR 95g:65146

3. I. Babuška and J.M. Melenk, The partition of unity method, Int. J. Numer. Meth. Eng. 40 (1997), 727-758. MR 97j:73071

4. I. Babuška and A. Miller, A feedback finite element method with a posteriori error estimation: Part I. The finite element method and some basic properties of the a posteriori error estimator, Comput. Methods Appl. Mech. Engrg. 61 (1987), 1-40. MR 88d:73036

5. I. Babuška and J. E. Osborn, Finite element-Galerkin approximation of the eigenvalues and eigenvectors of selfadjoint problems, Math. Comp. 52 (1989), 275-297. [MR 89k:65132]

6. I. Babuška and M. Suri, The $p$ and $h-p$ versions of the finite element method, basic principles and properties, SIAM Review 36 (1994), 578-632. MR 96d:65184

7. I. Babuška and M. Vogelius, Feedback and adaptive finite element solution of one dimensional boundary value problem, Numer. Math. 44 (1984), 75-102. [MR 85k:65070]

8. S.N. Bernstein, On the inverse problem of best approximation of continuous functions, Sochineniya II (1938), 292-294.

9. R. DeVore and F. Richards, Saturation and inverse theorems for spline approximation, in Spline Functions and Approximation Theory, A. Meir and A. Sharma, eds., Birkhäuser Verlag, 1973, pp. 73-82. MR 51:8698

10. J. R. Higgins, Completeness and Basis Properties of Sets of Special Functions, Cambridge University Press, New York, 1977. MR 58:17240

11. J. Nitsche, Ein Kriterium für die Quasi-Optimalität des Ritzschen Verfahren, Numer. Math. 11 (1968), 346-348. MR 38:1823

12. L.A. Oganesyan, and L.A. Rukhovets, Investigation of the convergence rate of variationaldifference schemes for second order elliptic equations in a two dimensional domain with a smooth boundary, (English translation) U.S.S.R. Comput. Math. Phys. 9 (1969), no. 5, 158183. MR 45:4665

13. P. Oswald, Personal Communication.

14. A. Pinkus, $n$-Widths in Approximation Theory, Springer-Verlag, Berlin, 1985. MR 86k:41001

15. A Schatz and J. Wang, Some new error estimates for Ritz-Galerkin methods with minimal regularity assumptions, Math. Comp. 65 (1996), 19-27. MR 96d:65190

16. A.F. Timan, Theory of approximation of functions of a real variable, Macmillian, New York, 1963. MR 33:465

Texas Institute for Computational and Applied Mathematics, University of Texas At Austin, Austin, TX 78712

Department of Mathematics, University of Maryland, College Park, MD 20742

E-mail address: jeo@math.umd.edu 